\title{
La tiflotecnología en la crisis de la covid
}

\section{Aids and appliances for blind people during the covid crisis}

\section{Moreno Montero ${ }^{1}$}

\section{Resumen}

Los 25 instructores de tiflotecnología y braille (ITB) de la ONCE que durante el confinamiento estuvieron atendiendo las demandas de las personas afiliadas sobre el acceso a la tecnología, analizan la experiencia, el desarrollo de las actividades, los recursos, los nuevos entornos y fórmulas de trabajo, así como su repercusión en el posconfinamiento. La transversalidad de la tiflotecnología se hizo patente en esos meses de confinamiento, en los que los ITB, desde el comienzo del estado de alarma hasta los actuales días de posconfinamiento, han tenido que actualizarse rápidamente en las nuevas herramientas y recursos tecnológicos que tanto usuarios como otros profesionales que intervienen en los procesos de atención (educativos, laborales, vida diaria, ocio y tiempo libre) necesitan, aplicando nuevas metodologías para facilitar su uso accesible. Los propios recursos técnicos, la autoformación, el trabajo en equipo, así como la coordinación con los diferentes profesionales o gestores que estaban en activo en ese periodo son aspectos fundamentales que han hecho posible la respuesta a las nuevas necesidades.

\section{Palabras clave}

Tiflotecnología. Atención remota. Teletrabajo. E-learning. Plataformas de comunicación. Autoformación.

\footnotetext{
$1 \mathrm{Ha}$ colaborado en la redacción de este artículo el equipo de técnicos en tiflotecnología y braille que estuvieron en activo durante el confinamiento de 2020: N. Santorum, L. Apastegui, F. Sierra, J. A. Giménez, Y. Tejada, M. Canadell, F. Albiar, P. Vilariño, R. Malvar, J. M. Fernández, J.M. López, M. Á. Alegría, R. Macías, J. Rodríguez, C. Meroño, V. Septién, P. Flores, M.S. Vargas, P. Roldán. M. Á. Fernández, Á. A. Mejías, M. Valentín, J. C. Carlos y M. Martín.
} 


\begin{abstract}
ONCE's 25 aids and appliances and braille counsellors who during lockdown responded to affiliates' requests for access to technology analyse their experience, performance, resources, new environments and working procedures and the respective post-lockdown impact. Lockdown highlighted the cross-disciplinary nature of aids and appliances for people with visual disability. Between the time the state of emergency came into effect and this writing, resources have had to be rapidly upgraded to deliver the new technological tools needed by both users and professionals. New methodologies have had to be deployed to enhance accessibility to support for educational, workplace, daily life and leisure time activities. Those new needs could be met thanks to the technical resources themselves, together with selftraining, teamwork and coordination among professionals or agents in different fields.
\end{abstract}

\title{
Key words
}

Aids and appliances for visually impaired persons. Remote care. Remote working. E-learning. Communication platforms. Self-training.

\section{Introducción}

La pandemia y, especialmente, el confinamiento han cambiado los hábitos y la forma de vida. En esta etapa en la que los contactos sociales y personales han sido imposibles o han estado restringidos, el uso de las Tecnologías de la Información y las Comunicaciones (TIC) ha sido crucial. Muchas personas con escasos o nulos conocimientos sobre ordenadores y dispositivos móviles se han visto abocadas a ampliarlos o iniciarlos para solventar o, al menos, paliar el problema del aislamiento.

Las tecnologías han posibilitado el contacto social, han permitido continuar con las actividades laborales y educativas, y se han consolidado como un importante recurso con el que llenar, incluso enriquecer, el tiempo libre.

El uso de la tecnología resulta más complejo cuando es imprescindible contar con información sobre entornos accesibles y con la instalación de las adaptaciones adecuadas al resto visual y a las tareas a desempeñar. La tiflotecnología es el conjunto de técnicas, conocimientos y recursos dedicados a procurar a las persones con ceguera o discapacidad visual grave los medios oportunos para una correcta utilización de la tecnología. En este

Moreno, I. (2021). La tiflotecnología en la crisis de la covid. RED Visual: Revista Especializada en Discapacidad Visual, 77, 109-138. https://doi.org/10.53094/MKQU7448. 
sentido, la función de los instructores de tiflotecnología y braille (ITB) es la de asesorar y formar a los afiliados sobre las ayudas y dispositivos técnicos adaptados que mejor se ajusten a la condición visual, intereses y necesidades. Estas tareas se realizan partiendo de una valoración específica de cada usuario, con especial incidencia en el ámbito educativo, laboral y cultural, sin olvidar la importancia del ocio y la ocupación del tiempo libre.

La pandemia ha supuesto un reto para muchos usuarios no iniciados en el uso de las tecnologías y para los ITB, especialmente, los que continuaron esta labor durante el confinamiento.

Debido a la dispersión de población en algunas comunidades autónomas, muchos afiliados ya estaban acostumbrados a la atención en remoto, sobre todo estudiantes o usuarios de ordenadores, permitiendo realizar formaciones a distancia debidamente planificadas, aunque siempre existía contacto presencial previo a fin de valorar o configurar cualquier cuestión.

El parón repentino durante los meses de confinamiento hizo replantearse varios aspectos, procurando que los usuarios estuviesen comunicados, informados y en permanente contacto para continuar o iniciar nuevas formaciones, utilizando cualquier medio disponible.

Coordinación, creatividad, imaginación, inventiva y, sobre todo, paciencia fueron calificativos muy acertados que definieron el periodo de confinamiento, en el que, por suerte, se pudo contar con diversos medios humanos o técnicos, buscando siempre la máxima implicación.

También otras iniciativas puestas en marcha con anterioridad en los centros de la ONCE resultaron vitales para mantener el contacto o promover iniciativas (como un Taller apoyo móviles, con más de 70 afiliados previamente instruidos por los ITB, que, vía WhatsApp, elaboraron diversos tutoriales en audio que permitieron utilizar nuevas plataformas como Zoom o Jitsi Meet).

Si resiliencia es la capacidad de adaptación de un ser frente a una situación adversa, está claro que la situación de trabajo durante el confinamiento por la covid-19 requirió de dicha capacidad.

No había tiempo para disfrutar de la vida personal y familiar, sin poder realizar ejercicio físico al aire libre, salir a un restaurante, tomar una copa con los amigos, etc. Fue una situación nueva a la que hubo que acostumbrarse. 
El miedo es una herramienta que ayuda a adaptarse a los cambios, más allá de las reacciones, que lleva a actuar y hacer frente a lo que provoca. Cada persona vive los acontecimientos de diferente forma, unos con miedo y con estrés, lo que crea ansiedad, rabia, molestias y agotamiento; otros lo viven de forma positiva, manteniendo activas las alertas para resolver la situación que atemoriza, lo que genera ganas de superar los retos.

Esta situación, con solo 25 profesionales para toda España de los 80 ITB de que dispone la plantilla de la ONCE a nivel nacional, con el soporte del Departamento de Autonomía Personal y del Centro de Tiflotecnología e Innovación (CTI), requirió la dedicación de largas jornadas laborales, cargadas de tareas y actividades que conllevaron una atención plena.

Hubo que asumir el cambio y, de alguna forma, reinventarse. Hubo que aprender a innovarse cada día, a tomar decisiones en grupo, compartir los miedos, las inseguridades y por supuesto, la confianza. Lo más difícil fue superar los retos y decidir que sí se podían afrontar los problemas ante esta situación, buscando alternativas de manera conjunta (creación de un equipo en Microsoft Teams para exámenes de la UNED, por ejemplo), guías didácticas de nuevas aplicaciones, lista de correo para comunicación entre los 25 ITB, etc.

Entendemos que lo que ha ocurrido no nos lleva a la normalidad, sino que estamos viviendo una transformación que ha venido para quedarse y a instaurarse en nuestras vidas.

\section{Contextualización y datos de intervenciones}

La pandemia provocada por la covid-19 golpeó a toda la sociedad, pero, muy especialmente, a los grupos más vulnerables, $y$, entre ellos, a las personas con ceguera, con baja visión o con sordoceguera, y también a la ONCE, en su doble vertiente: empresarial y prestadora de servicios sociales.

Desde el punto de vista institucional, se presentaron varias dificultades que afectaron de manera directa al colectivo de los ITB.

El confinamiento obligó a la ONCE a parar toda su actividad comercial. Pero, para la atención a las personas afiliadas, se adoptaron las medidas oportunas 
para cubrir las más urgentes y necesarias demandas. En materia de tiflotecnología y tecnología, se crearon equipos de guardia en el CTI y en Accesibilidad ONCE, pudiendo, además, contactar con el Centro de Atención al usuario (CAU) de Ibermática para el apoyo sobre estas cuestiones y, en los centros de la ONCE, se mantuvieron 25 puestos de instructores en tiflotecnología y braille con el $70 \%$ de jornada efectiva, de un total de 80 , distribuidos entre toda la geografía española.

La forma de atención se tuvo que modificar, y se pasó de la eminentemente presencial a las fórmulas a distancia, siendo las atenciones remotas las que representan el dato más significativo para la intervención de los ITB (Tabla 1).

Tabla 1. Atenciones remotas en 2020

\begin{tabular}{|l|r|}
\hline \multicolumn{1}{|c|}{ Mes } & \multicolumn{1}{|c|}{ Total } \\
\hline Enero & 188 \\
\hline Febrero & 217 \\
\hline Marzo (el 14 empieza el confinamiento) & 625 \\
\hline Abril (25 ITB desde el 5 de abril) & 670 \\
\hline Mayo (25 ITB) & 876 \\
\hline Junio (toda la plantilla desde el 15) & 662 \\
\hline Julio & 585 \\
\hline Agosto & 300 \\
\hline Septiembre & 515 \\
\hline Octubre & 591 \\
\hline Noviembre & 570 \\
\hline Diciembre & $\mathbf{3 8 3}$ \\
\hline Total & $\mathbf{6 1 2}$ \\
\hline
\end{tabular}

A continuación, se muestra gráficamente el número de intervenciones realizadas entre los meses de enero a diciembre de 2020. En la Figura 1 se puede leer el detalle numérico de cada uno de los meses. 
Figura 1. Diagrama de barras de atenciones remotas en 2020

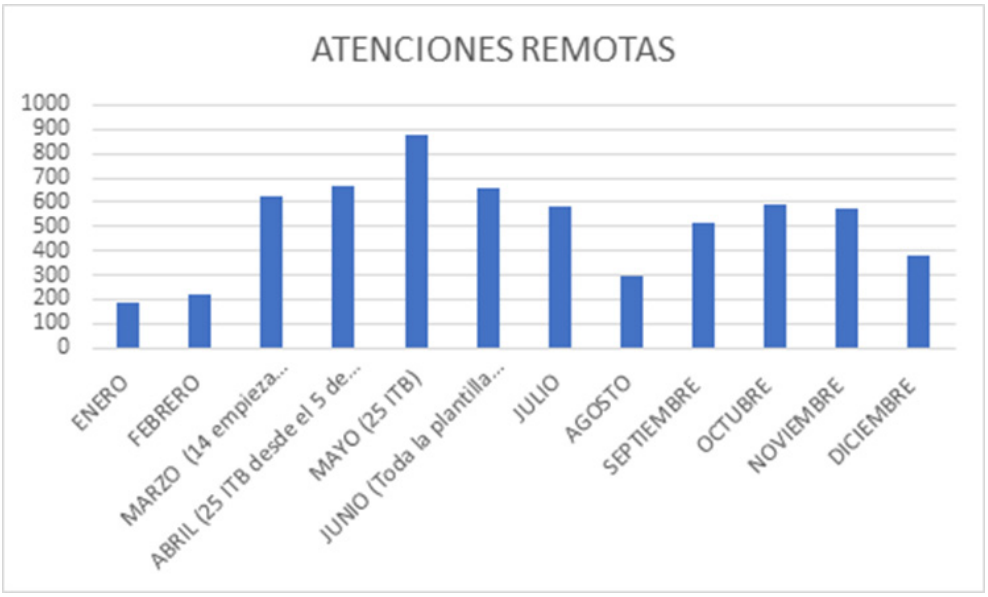

Por un lado, se observa un incremento del uso de sistemas remotos de intervención durante el periodo de confinamiento. En el mes de marzo se cuadruplican los datos de intervenciones realizadas, si se comparan con los de enero o febrero, y en mayo se multiplican por cinco.

Por otro, se inicia un leve descenso cuando se recupera la atención presencial en los meses de julio y agosto. En este descenso también ha influido el carácter estacional de estos meses. No obstante, si se comparase con los mismos meses del año anterior, podríamos observar que están por encima las cifras de atención del verano de 2020.

Por último, el incremento parece mantenerse. Se recupera en septiembre y, a partir de ese mes, cualquier otro duplica las atenciones de primeros de año, lo que hace suponer que este modelo de intervención remota parece haber venido para quedarse.

Asimismo, la necesidad de ocio y ocupación del tiempo libre que generó el confinamiento provocó un aumento significativo del uso de la Biblioteca Digital ONCE (BDO) y de la Videoteca Audesc (técnica audiodescriptiva). Desde estos dos servicios, las personas afiliadas pueden acceder a la lectura de libros (sonoro o braille) o a la reproducción de películas con audiodescripción.

Otro factor por destacar sobre su incremento fue la divulgación que se realizó desde el colectivo de los ITB y desde el resto de profesionales o centros (CTI, maestros, trabajadores sociales, gestores de departamento, voluntarios...). 
En el segundo trimestre del año aumentó el uso de los libros Daisy (Digital Accesible Information System) en un 45\%, y los TLO (formato de libro electrónico que la ONCE utiliza para poner a disposición de sus afiliados los libros que se han transcrito o producido en braille) en un $80 \%$ con respecto al trimestre anterior. La descarga de obras de Audesc tuvo unos aumentos importantes (147\%) y la visualización online alcanzo un $165 \%$.

Estos valores, al llegar el mes de junio, no dejaron de aumentar. Se han convertido en recursos de ocio valorados por el colectivo y, sobre todo, para los afiliados que se han incorporado al uso de esos servicios y que anteriormente no los utilizaban (Tabla 2).

Tabla 2. Descargas de la BDo y de Audesc en 2020

\begin{tabular}{|l|r|r|r|}
\hline \multicolumn{1}{|c|}{ BDO } & 01-01 AL 13-03 & 14-03 AL 15-06 & 16-06 AL 31-12 \\
\hline Partitura & 243 & 301 & 1093 \\
\hline Daisy & 76465 & 110620 & 223861 \\
\hline TLO & 3652 & 6563 & 10850 \\
\hline Total & $\mathbf{8 0 3 6 0}$ & $\mathbf{1 1 7 4 8 4}$ & $\mathbf{2 3 5 8 0 4}$ \\
\hline \hline Audesc & $\mathbf{0 1 - 0 1 ~ A L ~ 1 3 - 0 3 ~}$ & $\mathbf{1 4 - 0 3 ~ A L ~ 1 5 - 0 6 ~}$ & $\mathbf{1 6 - 0 6} \mathbf{A L} \mathbf{3 1 - 1 2}$ \\
\hline Descarga audio & 8378 & 20665 & 29137 \\
\hline Visualización online & 14910 & 39557 & 54163 \\
\hline Total & $\mathbf{2 3 2 8 8}$ & $\mathbf{6 0 2 2 2}$ & $\mathbf{8 3 3 0 0}$ \\
\hline
\end{tabular}

En la Figura 2 se muestra gráficamente el número de descargas de partituras, Daisy y TLO que hubo en el primer y segundo trimestre del año 2020 y en el segundo semestre.

Figura 2. Diagrama de barras de descargas de la BDO en 2020

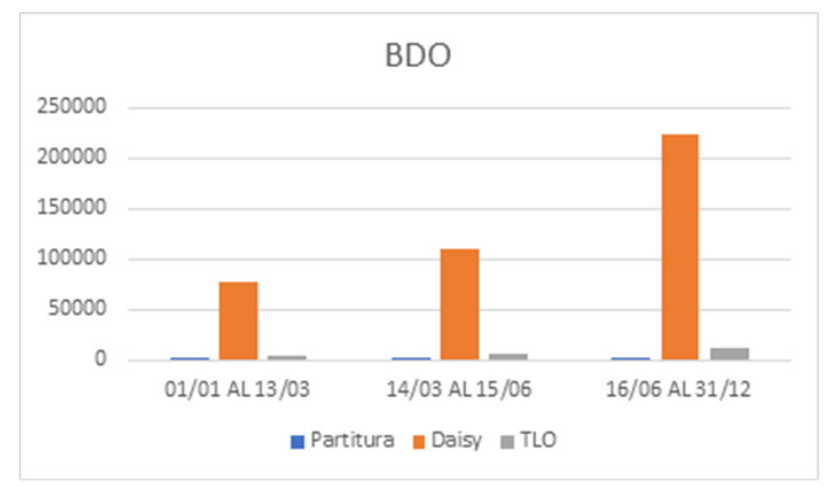

Moreno, I. (2021). La tiflotecnología en la crisis de la covid. RED Visual: Revista Especializada en Discapacidad Visual, 77, 109-138. https://doi.org/10.53094/MKQU7448. 
En la Figura 3 se muestra gráficamente el número de descargas de audio y de visualizaciones online de películas audiodescritas que hubo en el primer y segundo trimestre del año 2020 y en el siguiente semestre.

Figura 3. Diagrama de barras de descargas de Audesc en 2020

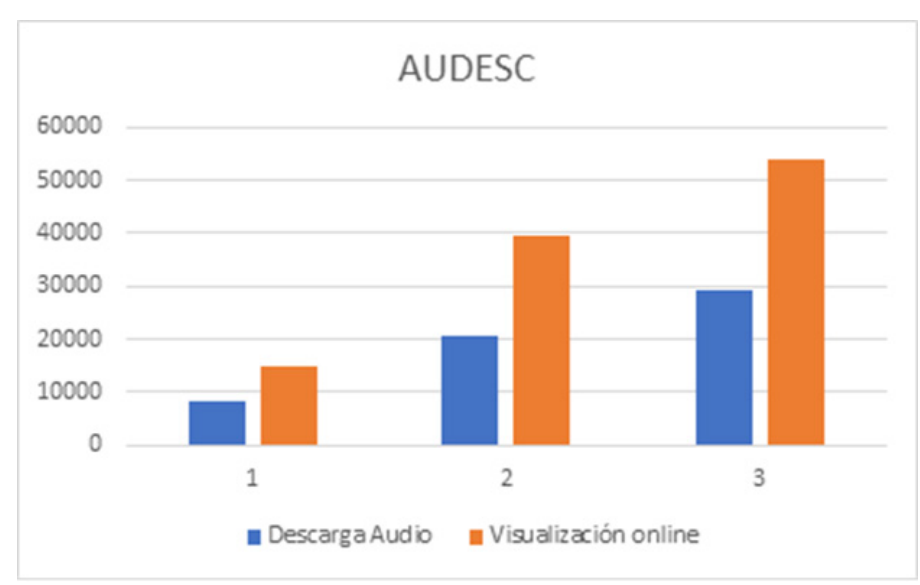

Finalmente, hay que señalar que las atenciones realizadas desde el 5 de abril al 15 de junio de 2020, por parte de los 25 ITB (con $30 \%$ de su jornada en ERTE), fueron algo superior a las 7000 .

\section{Importancia de la coordinación, el trabajo en equipo y la divulgación del servicio}

Habitualmente, la coordinación entre los diferentes actores que intervienen en Servicios Sociales para Afiliados resulta esencial para la consecución de objetivos comunes. Sin embargo, las especiales circunstancias que marcaron el período de confinamiento, pusieron de manifiesto hasta qué punto es importante en los procesos disruptivos y de adaptación.

La tiflotecnología destacó como pieza clave en un engranaje formado por un equipo multidisciplinar que incluía miembros de todos los centros y de diferentes categorías profesionales.

No obstante, centrando el análisis en la atención tiflotécnica, y teniendo en cuenta, como se explicaba anteriormente, que, del total de la plantilla de los ITB, solo per- 
manecieron en activo 25 técnicos con el $70 \%$ de la jornada, las funciones propias de la categoría profesional tuvieron que limitarse. Por ese motivo, quedó marcada como

[...] actuación fundamental la atención de incidencias y la prestación de soporte en la realización de tareas de apoyo a las TIC, priorizando los servicios laborales y de estudio, y garantizando el acceso a la comunicación y el ocio, para evitar aislamiento y mejorar la situación de colectivos especialmente vulnerables. ${ }^{2}$

Básicamente consistió en:

- Facilitar el acceso a plataformas educativas y a herramientas o aplicaciones para teletrabajo $^{3}$ y a aplicaciones o páginas web de necesidades básicas (sanitarias, compras, etc.).

- El soporte en la instalación de software y hardware tiflotécnicos, en propiedad o como adaptación al puesto de estudio (APE) o adaptación al puesto de trabajo (APT).

- El acceso y utilización del ClubONCE, la descarga de libros o películas, descarga de documentos educativos, etc.

- La configuración de herramientas tiflotécnicas y cualquier otra necesidad que surgiera y pudiera ser atendida de forma telemática relacionada con las TIC.

Ha de tenerse en cuenta que, a 31 de marzo de 2020, la ONCE contaba con un total de 72052 personas afiliadas, de las cuales: ${ }^{4}$

- 7273 eran estudiantes, incluyendo todas las etapas formativas. El 52,22\% de ellos (3798) se encontraban cursando educación obligatoria (de 3 a 16 años).

- 3746 eran activos laboralmente, con una ocupación distinta a la venta de productos de lotería. Un $28 \%$ de ellos en el Grupo Social ONCE y un $72 \%$ en empresas y entidades externas.

- 16396 pertenecían a colectivos especialmente vulnerables (13404 mayores de 55 años que vivían solos, más 2992 afiliados con sordoceguera).

2 Extracto de las funciones comunicadas por el Dpto. de RR. HH. para el período en ERTE.

3 Trabajo que una persona realiza para una empresa desde un lugar alejado de la sede de esta (habitualmente su propio domicilio) por medio de un sistema de telecomunicación.

4 Datos facilitados por los departamentos de Afiliados, Atención Educativa y Apoyo al Empleo.

Moreno, I. (2021). La tiflotecnología en la crisis de la covid. RED Visual: Revista Especializada en Discapacidad Visual, 77, 109-138. https://doi.org/10.53094/MKQU7448. 
Por todo ello, la ratio de afiliado/profesional condicionó en gran medida el volumen de atención y la distribución de responsabilidades. Sin olvidar que fue necesario atender a otros profesionales no afiliados (maestros propios y externos, y técnicos de otras empresas) que precisaron asistencia y formación para afrontar las situaciones de teletrabajo e e-learning. ${ }^{5}$

\subsection{Coordinación}

Para llegar a comprender el alcance de la coordinación a todos los niveles, resulta necesario agrupar las numerosas tareas realizadas en cada centro, a través de los diferentes profesionales y colaboradores.

\subsubsection{Detección de necesidades}

El contacto telefónico que se realizó durante todo el período de confinamiento permitió detectar la mayoría de las situaciones que precisaban atención urgente o que se encontraban en circunstancias de especial vulnerabilidad y aislamiento sin recursos.

En este proceso participó todo el personal que se encontraba fuera del ERTE, de manera total o parcial: directivos y responsables de servicio y gestión, miembros de los Consejos Territoriales, directores de Agencias, psicólogos, trabajadores sociales, especialistas en sordoceguera, personal administrativo, colaboradores del proyecto Oncerca y voluntariado. A través de numerosas llamadas diarias, se valoraba la situación específica de cada persona afiliada y se daban a conocer los servicios que la ONCE continuaba prestando a pesar de las dificultades. El objetivo era atender y acompañar a todos los afiliados de la organización.

\subsubsection{Priorización y distribución de atenciones}

Los gestores de Servicios para Afiliados y los de Autonomía Personal, principalmente, desarrollaron una complicada función de cribaje. Debían priorizar los casos que precisaban atención urgente y distribuir tareas entre los diferentes ITB en activo, o derivarlos a otro tipo de atención, como soporte psicológico o la asistencia a través de

\footnotetext{
5 Procesos de enseñanza-aprendizaje que se llevan a cabo a través de Internet, caracterizados por una separación física entre profesorado y estudiantes, pero con el predominio de una comunicación tanto síncrona como asíncrona, a través de la cual se lleva a cabo una interacción didáctica continuada.
} 
voluntariado, por ejemplo. Según la organización de cada centro, en ocasiones este cribaje se complementaba con la actuación de otros profesionales.

Se ha de destacar que todo el personal que participaba en estos procesos estaba sometido a las dificultades sobrevenidas por el cambio de funciones habituales que, además, debían realizarse a distancia, pero que iban superándose para la consecución de las demandas de las personas afiliadas.

\subsubsection{Servicio de tiflotecnología}

El servicio de tiflotecnología se desarrolló con 25 ITB que cubrieron las necesidades de accesibilidad tecnológica de todo el territorio nacional. La capacidad de improvisación fue esencial en un momento en el que la atención y las herramientas utilizadas habitualmente sufrieron un drástico cambio, demostrando así la cualificación como profesionales que dan respuesta a todos los problemas de ámbito tecnológico, con recursos y habilidades multidisciplinares, que permiten probar innovaciones, aplicarlas en el ámbito educativo, laboral o doméstico e instruir en su utilización.

No obstante, las dificultades derivadas del nuevo rol del ITB conllevaron una enorme tarea de organización y coordinación de todo el equipo, con el objetivo de conseguir soporte recíproco, para poder afrontar la multitud de situaciones que se dieron esos días. No solo con las diferentes plataformas de comunicación, sistemas operativos, dispositivos y adaptaciones, sino, también, con la gran variedad de usuarios atendidos.

A la gravedad de la situación se le sumaron otras dificultades, como, por ejemplo, la falta de material especializado y recursos en algunos centros, que quedaron almacenados en las aulas de tiflotecnología, sin posibilidad de acceder a ellos. Cosa que pudo subsanarse con la asistencia entre compañeros y la gran ayuda que supusieron los especialistas del CTI en activo, así como la disponibilidad de los recursos materiales personales de los ITB o sus entornos familiares.

\subsection{Trabajo en equipo}

El desarrollo del servicio de tiflotecnología precisó una gran coordinación, llevándose a cabo a través de las siguientes estrategias: 
a. Comunicación. A nivel general, se adaptó la red de contactos y herramientas. Se creó una lista de distribución en correo electrónico con los profesionales en activo y se comenzaron a utilizar nuevas plataformas de videoconferencia (Zoom, Google Meet, Jitsi Meet, Microsoft Teams). Una comunicación fluida permitió la rápida solución de dudas e incidencias y, sobre todo, la colaboración continua. Cada centro estableció su propia red de contactos para la distribución de tareas y optimización de la atención territorial.

b. Recursos compartidos. Se estableció una carpeta compartida específica para recursos didácticos, que fueron creándose al ser necesarios para el desarrollo de la actividad, y se mantuvo el uso de la carpeta compartida desde Accesibilidad ONCE que contiene recursos de software (scripts, drivers, instrucciones técnicas, ejecutables...) para la atención a las personas afiliadas que, sobre todo, trabajan en el entorno de la ONCE.

c. Formación y experimentación. El teletrabajo y las nuevas herramientas precisaron continuas evaluaciones de accesibilidad y numerosas pruebas para conseguir que, tanto técnicos como usuarios, pudieran aplicarlas a sus tareas laborales, académicas, culturales y de ocio. Con las aportaciones de todo el equipo, se elaboraron guías didácticas y manuales que facilitaron la formación directa de afiliados, la de otros técnicos en activo y la posterior preparación de todos los ITB una vez finalizó el ERTE.

d. Nuevas estrategias. Las especiales circunstancias del período de confinamiento requirieron la aplicación de nuevas estrategias de atención, que debía ser totalmente virtual. La asistencia remota con JAWS Tandem, NVDA Remote o TeamViewer (herramientas de asistencia remota para uso de los ITB afiliados y no afiliados) se complementó con el uso de mensajería instantánea, llamadas telefónicas, videollamadas y plataformas de videoconferencia. También se elaboraron guías y tutoriales en diferentes formatos multimedia y se promocionó su acceso a través de las webs corporativas, blogs, web didácticas y canales de difusión en YouTube que, además, incluyeron numerosas actividades culturales y de entretenimiento.

Se ha de destacar que estas nuevas estrategias siguen utilizándose y mejorando actualmente, ya que supusieron un cambio sustancial en la atención tiflotécnica que ha permitido continuar con el servicio a pesar de las restricciones de atención y movilidad en todo el estado. 
e. Soporte. El más que necesario soporte mutuo de todo el equipo de ITB en activo durante el período de confinamiento también precisó la inestimable colaboración del personal y de los técnicos del CTI y de la Dirección Técnica de Sistemas y Tecnologías de la Información, maestros y de todos los demás profesionales que facilitaron atenciones e instalaciones, resolución de incidencias y la gestión de materiales de adaptación.

No menos importante fue la colaboración que se encontró en profesionales externos $y$, sobre todo, en los propios afiliados, familiares e incluso vecinos, que facilitaron en los momentos más difíciles conectar remotamente para poder realizar la atención. Por poner un ejemplo, los casos de personas con ceguera que vivían solas, con equipos informáticos sin la adaptación precisa o que no acababan de funcionar y que, gracias a sus intervenciones, pudieron lograrse superar las dificultades para conseguir los objetivos necesarios.

Cabe determinar entonces que el positivo resultado de la atención realizada durante todo el período de confinamiento fue consecuencia directa de la coordinación y trabajo en equipo de todos los profesionales implicados y de las personas del entorno de los afiliados (familia, vecinos, voluntarios...).

\subsection{Divulgación del servicio}

En todo este período cobra especial relevancia la difusión de las acciones llevadas a cabo, no solo para que la población en general conozca la importante labor social que la ONCE desarrolla, sino también para llegar a todos los afiliados y afiliadas.

Se emitieron algunos reportajes y cuñas publicitarias en diferentes espacios de ámbito nacional y autonómico, como, por ejemplo:

- Espacio diario de Sorteos ONCE en TVE del día 16 de mayo de 2020, en el que se menciona la importancia de la Red para facilitar el acceso de las personas ciegas a entornos educativos, laborales, de cultura, ocio o información, y se destaca la necesidad de contar con plataformas accesibles y los beneficios de la tiflotecnología. Disponible en: https://www.rtve.es/m/alacarta/videos/sorteos-once/once-16-05-20/5577105/.

- Programa Para que veas, de Patty Bonet, emitido en Radio 5 (de RTVE) el día 25 de mayo de 2020 con el título Día de internet: la accesibilidad abre el mundo a

Moreno, I. (2021). La tiflotecnología en la crisis de la covid. RED Visual: Revista Especializada en Discapacidad Visual, 77, 109-138. https://doi.org/10.53094/MKQU7448. 
los ciegos y su ausencia aísla, donde se hace un llamamiento sobre la necesidad de una accesibilidad plena y real de las personas ciegas o con discapacidad visual grave, para poder afrontar todo tipo de situaciones. Disponible en: https://www.rtve.es/alacarta/audios/para-que-veas/para-veas-dia-internet-accesibilidad-abre-mundo-ciegos-su-ausencia-aisla-25-05-20/5581926/.

- Cuñas publicitarias en radios nacionales y autonómicas, emitidas y repetidas en horarios de máxima audiencia, sobre la función social de la ONCE y la actividad de sus profesionales, con los lemas Gente con ilusión y Con esta actitud, todo saldrá bien.

- El Món a RAC1. Programa del 8 de mayo de 2020 (emitido a las 9:41:54). Descarga disponible en MP3 en: https://audioserver.rac1.cat/get/061b8844-08ad-47a8-ac8c-ca5717e44f45/1/2020-0508-el-mon-a-rac1-9h.mp3?source=WEB\&download.

- Herrera en COPE. Programa del 3 abril de 2020. Disponible en: https://www. cope.es/programas/herrera-en-cope/herrera-en-cope-hora-completa/audios/ herrera-cope-03-04-2020-20200403_1064758 (minuto 23:52 del archivo de audio).

- Informativo Telerioja de RTVE, del día 13 de mayo de 2020. Reportaje sobre estudiantes con discapacidad visual en La Rioja y el uso de la Tiflotecnología en la educación a distancia. Disponible en:

https://www.rtve.es/alacarta/videos/informativo-telerioja/informativo-telerioja-2-13-05-20/5575071/?t=07m20s.

\section{4. ¿Quiénes han sido los usuarios atendidos?}

El distanciamiento creado por el confinamiento generó múltiples dificultades que nos obligó a ser dinámicos, flexibles e imaginativos en la instrucción y resolución de problemas teniendo en cuenta las dificultades específicas de cada usuario.

Hay que destacar la necesidad y la importancia para los usuarios de obtener una autonomía y participación activa en su vida cotidiana. Sin embargo, no todos parten del mismo punto ni tienen los mismos recursos o capacidades para hacerlo de manera 
efectiva. Sus habilidades y competencias digitales, recursos utilizados y adecuación facilitan, en gran medida, el uso de la tecnología y el acceso a la misma.

Una tecnología no accesible supone una barrera mayor y un motivo para dificultar la integración de una persona con un déficit visual.

Gran parte de las atenciones se realizaron con personas mayores con el manejo e incidencias de la BDO y de las películas con audiodescripción en sus dispositivos móviles y ordenadores, con las que, en ocasiones, necesitábamos la ayuda de familiares, acompañantes, cuidadores y cualquier persona próxima que se prestara para su colaboración.

Otras atenciones que se prestaron mayoritariamente y de forma regular fueron a los estudiantes en diferentes etapas educativas, desde niños de primaria hasta adultos que cursaban estudios universitarios, así como a sus maestros de apoyo de la ONCE.

Los estudiantes de la Escuela Universitaria de Fisioterapia de la ONCE también recibieron apoyo en sus exámenes a través del Campus Virtual y con seguimiento a través de la herramienta Zoom.

Además, se mantuvieron reuniones con entidades como la UNED con el fin de mejorar la accesibilidad a la plataforma de exámenes. O con la administración pública, para la realización de pruebas de capacitación profesional a las que se presentaban personas afiliadas a la ONCE.

Los usuarios con sordoceguera fueron otro de los colectivos a los que se atendió durante el confinamiento. Inicialmente, se contó con la participación de sus mediadores y, posteriormente, debido a su ausencia, nos tuvimos que valer de la ayuda de familiares, amigos e incluso vecinos.

El contacto con los compañeros de los centros trabajo también fue continuo. Desde el principio del confinamiento se les prestó ayuda para realizar las conexiones remotas o el uso de las herramientas de comunicación.

El teletrabajo llegó para quedarse y obligó a todos a adaptarse a esta nueva forma de trabajar, facilitando la accesibilidad en el uso de herramientas para estos fines 
(conexiones VPN, escritorios remotos y virtuales, etc.), por lo que, muchas veces, hubo que contactar con el soporte informático de los usuarios y, en muchas ocasiones, establecer colaboraciones en conjunto con los técnicos del CTI o de la Dirección Técnica de Sistemas y Tecnologías de la Información de la ONCE para solventar problemas de accesibilidad con las aplicaciones o los equipos.

\section{Equipamiento técnico durante la pandemia}

La primera cuestión importante que le surge a un ITB es el tema de la diversidad de dispositivos, en lo que respecta tanto al hardware (ordenadores, móviles y tabletas), como al software (sistemas operativos, aplicaciones o programas) y la variabilidad de combinaciones entre ambos que pueden presentarse. Este despliegue de medios no tendría sentido sin un software accesible que permitiese controlarlos y explicarlos.

En este caso, y dado que la comunicación del confinamiento tuvo lugar durante el fin de semana, produjo una situación en la que la mayoría de los ITB tuvo que echar mano de herramientas y equipos propios, dado que los equipos corporativos se quedaron en las sedes de la Institución.

Además, para atender las crecientes demandas surgidas durante el confinamiento total, en muchos casos hubo que adaptarse a utilizar nuevas herramientas para explicar su uso al grueso de afiliados.

La segunda cuestión importante ante la falta de contacto presencial es la gran variedad de tipos de comunicaciones y la desigualdad en la calidad de estas, dado que no es lo mismo conectarse a un equipo cableado con fibra óptica, que permite grandes volúmenes de información, que a un dispositivo móvil con baja cobertura.

La tercera cuestión es el perfil de la persona, en este caso, afiliada que utiliza la tecnología. Hay mucha diferencia entre atender a una persona con ceguera total y a una persona con baja visón.

En el Apéndice se relaciona el equipamiento específico, en general, utilizado entre todos los ITB durante la pandemia. 


\section{Dificultades y soluciones}

Proporcionar una vía de acceso a la comunicación, al ocio, al estudio o al trabajo con el apoyo de las nuevas tecnologías supuso un reto no exento de dificultades. Mientras que antes, en muchas ocasiones, se solucionaban con una visita, una intervención directa o una atención presencial, en este periodo hubo que ir solventando numerosas y dispares situaciones con las posibilidades que nos ofrecen los medios técnicos, pero, sobre todo, con paciencia y mucho esfuerzo. El conjunto de dificultades encontradas y las soluciones planteadas se pueden englobar en los siguientes apartados.

\subsection{Disponibilidad}

La primera gran dificultad importante que generó la pandemia fue la conciliación laboral y familiar, intentando ajustar muchas situaciones nuevas: la suspensión de nuestra vida social, asumir nuevas rutinas dentro de nuestro hogar, las videoconferencias, llamadas, la gestión del teletrabajo, las necesidades familiares.

La sensación generalizada fue la de que el número de técnicos y el porcentaje de jornada resultaron insuficientes para atender una necesidad que surgió de forma excepcional. El $70 \%$ de la jornada se quedó corto, y se optó por la flexibilidad tanto en las horas como en el tiempo de atención. No se podía quedar una sola persona afiliada sin posibilidad de comunicación con el exterior, o sin nuevas oportunidades de ocio.

\subsection{Medios técnicos}

Los medios con los que se cuenta en el puesto de trabajo no son los mismos con los que se podía contar en los domicilios. Se pudo resolver de diferentes modos: en algunos casos, desplazando equipos al domicilio del técnico, pero, en la mayoría, aportando dispositivos propios.

También se utilizaron herramientas de trabajo que no habían sido utilizadas hasta ese momento, como es el caso de Zoom, Teams, Classroom, Meet, etc. Hubo que realizar un esfuerzo grande y en poco tiempo tanto de autoformación como de formación a familiares y otros profesionales.

Otra dificultad técnica propia del teletrabajo radica en la baja calidad de algunas conexiones, tanto propias como de los usuarios, que no tienen más solución que la

Moreno, I. (2021). La tiflotecnología en la crisis de la covid. RED Visual: Revista Especializada en Discapacidad Visual, 77, 109-138. https://doi.org/10.53094/MKQU7448. 
de dedicarle más tiempo a este tipo de intervenciones o bien posponerlas. En muchas ocasiones, la conectividad (wifi, 4G) o los dispositivos tecnológicos (smartphone, ordenadores y herramientas para el trabajo remoto) no eran lo suficientemente potentes para dar un servicio de calidad.

En otros casos, se detectaron carencias en la dotación tecnológica de los usuarios (no disponer de ordenador, tableta, smartphone...), o, si se tenían, tuvieron que compartirlos con el resto de la familia (padres teletrabajando, hermanos en otros niveles educativos...). La solución aportada fue la de buscar horas de atención por las tardes o en fines de semana.

\subsection{Falta de competencias digitales}

El confinamiento, la reclusión en casa..., han dejado al descubierto las carencias en cultura digital y en recursos tecnológicos de las familias en general y de los afiliados en particular. En cuestión de días, de vivir, estudiar y trabajar en el exterior del núcleo familiar se pasó a realizar estas actividades vitales en y desde el interior de nuestros hogares.

También las empresas y los centros educativos han mostrado carencias y dificultades de adaptación a la situación extraordinaria del confinamiento.

En cuanto a los usuarios, no todos cuentan con la capacitación básica para afrontar el aprendizaje de una nueva herramienta. Podemos destacar:

- Falta de alfabetización digital en algunos sectores.

- Desconocimiento de términos comunes hoy en día (nube, datos, almacenamiento...).

- Escasas habilidades en el manejo de la tecnología por parte de algunos usuarios.

Hay que tener en cuenta que la atención remota ${ }^{6}$ requiere mayores habilidades por parte de los usuarios que en las atenciones presenciales.

La falta de alfabetización digital se supone en dos perfiles de usuarios, como son las personas mayores - con una menor adaptabilidad a las nuevas tecnologías de manera

6 Se entiende por asistencia remota el servicio que permite la solución de problemas asistidos por un técnico utilizando internet y aplicaciones para poder realizar acceso remoto a los equipos del usuario al que se le presta la atención.

Moreno, I. (2021). La tiflotecnología en la crisis de la covid. RED Visual: Revista Especializada en Discapacidad Visual, 77, 109-138. https://doi.org/10.53094/MKQU7448. 
general-, así como algunas personas con sordoceguera, sobre todo personas sordas con ceguera sobrevenida que no han tenido el acceso a un vocabulario normalizado.

Las soluciones aplicadas en este aspecto han sido:

- La primera y fundamental, mucha paciencia.

- Explicación muy detallada de los pasos que conlleva cada procedimiento, transmitido verbalmente o enviado en forma de pequeños tutoriales.

- Coordinación con servicios técnicos.

- Apoyo de familiares, voluntariado y personas del entorno del usuario.

- Nueva forma de explicar. Al impartir sesiones telefónicas, fue muy importante incidir en el funcionamiento de cualquier adaptación sobre un sistema operativo: estructuras, interfaz gráfica, elementos, terminología...

\subsection{Limitaciones de las herramientas de atención remota}

No todas las atenciones pueden ser solucionadas de forma remota, requiriendo una intervención presencial. En estos casos, al no resultar posible, se fueron solventando, en la medida en que se pudo, fundamentalmente con apoyo de personas del entorno de los usuarios y con el uso de videoconferencias.

También ha incidido el hecho de que los ITB llevasen tiempo utilizando las herramientas de atención remota de manera regular. La experiencia es una receta que hemos podido aplicar, de forma que, en muchas ocasiones, al escuchar un mensaje de voz de un dispositivo ya sabemos en qué situación se encuentra este, aunque no se esté delante para comprobarlo.

\subsection{Fragmentación}

La diversidad de dispositivos, software, sistemas operativos, capas de personalización, etc., inciden en gran medida en nuestra manera de intervenir. Es evidente la diferencia entre la atención remota en un ordenador o un móvil. Se tuvieron que utilizar servicios propios y privados, como WhatsApp, y realizar videoconferencias. 
También existe el problema de los permisos de control por parte del usuario que nos debe habilitar, $y$, en otras ocasiones, son los técnicos informáticos los que deben habilitar ciertos permisos para el funcionamiento.

Esto, unido a la diversidad de usuarios, convierte el trabajo del ITB en un campo de pruebas ilimitado. Por ejemplo, es muy diferente realizar una sesión remota a un dispositivo iOS que a otro Android, unido a que, a veces, no es posible controlar el equipo (por ejemplo, en un reinicio tras una actualización).

\section{Anécdotas de la intervención}

La repentina irrupción del teletrabajo y la asistencia remota casi como única forma de intervención con el afiliado ha supuesto infinidad de situaciones inesperadas que se han ido sorteando con mayor o menor fortuna. Muchas de estas situaciones quedarán en el recuerdo como divertidas anécdotas, otras quizás no tanto, pero quedémonos con las primeras.

Un simple equívoco da lugar a un enredo, desesperante en el momento, pero divertido una vez solucionado: un usuario con su teléfono en modo avión, por lo que difícil contactar con él para atenderle si no intervienen los servicios sociales del lugar para que los vecinos solucionaran el entuerto; un niño afiliado que hace de intérprete de su padre, que no sabe español, para una instalación por videollamada; configurar una impresora braille para un niño afiliado siendo el interlocutor el abuelo con déficit auditivo y las consiguientes malas interpretaciones, o un «espontáneo» en formación online con nombre idéntico al del afiliado que se está atendiendo...

Imagine una situación en la que el usuario demanda aplicaciones de ocio para su teléfono que le permitan lectura de libros o disfrutar de películas audiodescritas, cosas que nunca ha visto pero de las que le han hablado. Hasta el momento, para él, un teléfono solo servía para realizar llamadas. Sucedió que un usuario hizo esta demanda y, cuando se contactó con él, no sabía decir qué teléfono poseía. No sabía si era Android o Apple. Pidiéndole que diera algún detalle que revelase el tipo de terminal, no se conseguía averiguarlo. Se le pide a un familiar que describa el aspecto de la pantalla para intentar identificar el terminal, la marca. La sorpresa llegó al describir que la pantalla solo mostraba la hora y que el teléfono era de teclas (imposible instalar una $a p p)$. 
Son muchas las ocasiones en las que las sofisticadas herramientas para hacer conexiones remotas con las que poder ver la pantalla del usuario no eran suficiente soporte para realizar una asistencia. En muchas ocasiones, hubo que tirar de cosas más rudimentarias, por llamarlas de alguna forma. Una simple videoconferencia por WhatsApp o FaceTime ha solucionado muchos problemas en los que el ITB necesita tener un par de ojos al otro lado de la conexión. Puede suponerse que intentar que una persona con ceguera haga foco sobre la pantalla con una videollamada no es tarea fácil. Ha sido así como se ha podido averiguar la contraseña de un rúter, discernir el modelo del lector de libros hablados que estaba usando, rastrear con una videollamada un cable de Ethernet que atravesaba toda la casa con varios empalmes hasta lograr averiguar cuál era el que estaba desconectado.

Sin embargo, la tecnología no siempre es suficiente y, a veces, la presencia física es imprescindible. El voluntariado en tiempos de pandemia se ha revelado como el gran aliado. Muchas han sido las veces en las que se han convertido en los ojos y manos de los ITB, siguiendo instrucciones telefónicas cuando los lectores de pantalla, los asistentes de voz o las líneas braille han dejado de funcionar. Mención especial para los mediadores de personas con sordoceguera, pues establecer un contacto telefónico previo para iniciar una sesión remota se hace imposible. Es necesario que alguien, al otro lado del teléfono, cuente lo que un usuario quiere decir cuando solo puede usar la dactilografía para comunicarse.

En el caso de los usuarios con sordoceguera, estos viven la pandemia de una manera significativa, ya que esta discapacidad supone superar muchas barreras para establecer comunicación con su entorno, tarea aún más difícil si añadimos el fenómeno del confinamiento. Esto puede verse reflejado en el caso de una usuaria sorda de nacimiento y ciega en los últimos años. Su pérdida de visión ya está tan agravada que decide comprar un iPhone y una línea braille para conectar a este. Tras un par de sesiones de las muchas necesarias para adquirir un manejo fluido de estos dispositivos, comienza el periodo de confinamiento, paralizando su formación, con tan mala fortuna que su línea braille comienza a dar fallos, desconectándose del iPhone con frecuencia. Ni ella ni su marido, también sordo, pueden resolver este problema técnico, debido a un mal funcionamiento del Bluetooth. La asistencia telefónica no es posible por motivos evidentes.

Gracias a la labor de sus mediadores se pudo establecer contacto entre ellos y el CTI para poder realizar un cambio por una nueva línea braille, que ya por fin funcionaba perfectamente sin desconexiones. 
El nuevo equipo se instaló y configuró a través de una videollamada de Skype para dar instrucciones al mediador de las operaciones de configuración. La sonrisa y los gestos de la usuaria fueron la mayor recompensa al esfuerzo de profesionales, mediadores, maestros, ITB..., que habían intervenido en su proceso de formación.

\section{Cambios en la atención tiflotécnica}

Antes de la vuelta a la actividad tras el confinamiento, se creó un grupo de trabajo con varios de los 25 ITB para analizar las circunstancias, las nuevas necesidades, los procesos y las metodologías, y se elaboraron una serie de documentos para las personas afiliadas y los profesionales con pautas y consejos para una intervención lo más segura posible.

Tras volver a la actividad, algunas cuestiones que antes eran menos habituales forman parte de una nueva normalidad:

- Aumento de las demandas de asesoramiento en compras y pagos online, plataformas de compras o contenidos multimedia, especialmente de servicios del ClubONCE, como la biblioteca digital de la ONCE (GOLD) y de audiodescripción (Apolo ONCE).

- Apoyos técnicos ajenos a la formación. Asistencias sobre problemas ajenos al servicio, como configuraciones, instalaciones, incidencias técnicas o gestión de licencias.

- Herramientas remotas y/o de comunicación online. Hicieron posible continuar formaciones con afiliados; sobre todo, afiliados con dificultades para acercarse a las aulas (por edad, transporte, distancia, confinamiento, etc..). También brindan nuevas posibilidades, ofreciendo colaboración entre compañeros y una mayor divulgación de la tiflotecnología. Aumento de actividades, reuniones, charlas mediante diferentes sistemas de videollamada (Zoom, Skype, etc..).

- Aumento del trabajo colaborativo.

- Aumento de la petición de asesoramiento en la accesibilidad de sistemas telemáticos: web, app, etc. 
- Formación general. La importancia de contar con conocimientos básicos sobre nuevas tecnologías es vital. Redes, comunicación, información, seguridad, protección de datos..., están en juego hoy más que nunca.

- Incorporación de las medidas de higiene en la atención presencial (abrir las ventanas, utilización de gel hidroalcohólico, desinfección de equipos y mobiliario con viricidas, etc.) siguiendo las normas establecidas al respecto.

- Adaptación de los diferentes servicios anteriormente establecidos para que se puedan realizar de manera telemática:

- Elaboración y actualización, por parte de la ONCE, de la página web de ClubONCE con nuevos contenidos:

- Tutoriales de plataformas (Zoom, Teams, Meet...).

- Tutoriales para la descarga de libros y visualización de películas.

- Demanda de servicios (servicios sociales, vendedores afiliados, etc.).

- Nueva página del CTI. Con venta online, catálogo, venta de segunda mano, recursos formativos (guías y manuales), etc.

- Mayor implicación de voluntarios y/o familiares en la facilitación a los afiliados del acceso de servicios telemáticos.

\section{Conclusiones}

Desde marzo de 2020 hasta ahora nos está tocando vivir en un contexto muy atípico debido a la pandemia. Por este motivo, han surgido nuevas necesidades o dificultades para el acceso de las personas con discapacidad visual a las tecnologías, habida cuenta del incremento de estas en la sociedad en la crisis provocada por la covid.

El uso de la tecnología, en general, supone para las personas con discapacidad un arma de doble filo. Por un lado, proporciona nuevas herramientas que posibilitan tareas que no podían realizarse sin ella y, por otro lado, al no cumplirse, en muchas ocasiones, las pautas o normas de accesibilidad en el diseño de las aplicaciones o 
dispositivos, no se garantiza el uso normalizado de la tecnología a personas con baja visión, ceguera o sordoceguera.

El acceso a la tecnología es un derecho fundamental, y garantizar su acceso a las personas con discapacidad visual es primordial para su plena participación en la sociedad. Desde participar en una conferencia hasta asistir a clase o consultar las cuentas bancarias.

Y no hay un menú igual para todos, y nos debemos adecuar a las necesidades de los usuarios y sus particularidades. La importancia de la tecnología para la comunicación, la formación, la actividad laboral, el ocio y la cultura, y la adaptación a situaciones extraordinarias. Para ello, es necesario incidir en la capacitación digital para estar preparados.

No se puede obviar un hecho importante: una persona con ceguera o con baja visión capacitada tecnológicamente puede realizar prácticamente las mismas tareas que una persona sin discapacidad visual.

En la sociedad del conocimiento en la que nos encontramos, son muchas las situaciones en la vida de una persona que pasan por el uso de la tecnología. Leer un libro digital, hacer la compra semanal, mantener comunicación con un grupo de amigos, gestionar citas médicas o las cuentas bancarias, desarrollar un trabajo profesional, etc. $Y$ todo ello, interconectándose desde puntos geográficamente lejanos, en un abrir y cerrar de ojos, gracias a la red de Internet.

La transversalidad de la accesibilidad a la tecnología y, por ende, de la tiflotecnología, era un hecho que tomó especial relevancia en el confinamiento y que ha persistido tras el mismo. Más que nunca, se ha puesto en relieve la cara y la cruz de esta situación. Sin las plataformas de teletrabajo, los campus virtuales, la compra online, los pagos desde el móvil, las aplicaciones para mantener reuniones privadas y profesionales y un largo etcétera, se habría aislado y paralizado, en mayor medida, el mundo en el que vivimos.

Podemos concluir que creemos que hemos tenido una experiencia profesional durante el confinamiento complicada, ardua y difícil, pero que nos ha servido para poder darnos a conocer a muchos afiliados de nuestra Organización que no tenían conocimiento de nuestra existencia. 
Asimismo, nos ha servido, de una manera gratificante, para poder realizar todas las intervenciones que tuvimos durante el confinamiento y poder ayudar a tantos y tantos afiliados de nuestra Organización. Se ha conseguido:

- Que una niña pueda estudiar desde casa como el resto de sus compañeros de clase.

- Que el universitario realice, con garantías, los exámenes del grado.

- Que el profesional teletrabaje con las medidas y recursos accesibles como el resto de los trabajadores de su empresa.

- Que la persona con sordoceguera pueda realizar la compra online de lo que necesita cada día, ella y su familia.

- Que los mayores que han tenido que quedarse en casa o en la residencia puedan ocupar su tiempo leyendo o informándose de lo que sucede en el mundo cada día.

Y a pesar de que el número de profesionales que quedó en atención durante el ERTE fue inferior al que se necesitaba - dada la situación que se generó de convergencia de un número elevado de demandas con la rapidez y la urgencia en la respuesta, más la trascendencia de muchas de ellas, al cubrir necesidades de importancia para las personas afiliadas u otros profesionales que debían atenderles, como los maestros-, cabe determinar que el positivo resultado de la atención realizada durante todo el período de confinamiento fue consecuencia directa de la coordinación y trabajo en equipo de todos los profesionales implicados e, incluso, del apoyo incondicional de los compañeros ITB que estaban en ERTE.

Todos y todas, usuarios, profesionales, familiares, voluntarios, etc., hemos tenido que aprender muy rápidamente nuevas aplicaciones, herramientas o plataformas, adaptarnos a distintos entornos y formas de trabajo o estudio y su accesibilidad, para poder afrontar el confinamiento y, tras él, poder seguir superando las barreras que la pandemia nos presenta en el día a día.

Y lejos de poner en alto el papel que ha desempeñado la tiflotecnología en esta crisis de la covid, todos y cada uno de los ITB que hemos participado de esta experiencia creemos que, a pesar de las dificultades y el duro trabajo, lo trascendente y relevante ha sido cada una de las personas que han hecho todo posible: desde el que ha ayu- 
dado hasta el que ha resuelto los problemas, desde el que ha enseñado hasta el que ha aprendido, desde el que ha detectado las necesidades hasta el que ha gestionado los recursos para cubrirlas, porque, a pesar del distanciamiento físico, muchos nos hemos sentido presentes y unidos para poder hacer posible lo que parecía imposible.

Ignacia Moreno Montero. Instructora de Tiflotecnología y Braille. Delegación Territorial de la ONCE. Prim, 3, 28004; Madrid (España).Correo electrónico: igmm@once.es. 


\section{Apéndice. Herramientas utilizadas por los ITB}

\section{Hardware}

1. Anotadores y líneas braille

a. Líneas braille Focus: Focus 14 Blue (V Generación), Focus 40 y Focus 14 Blue. Se utilizaron varios equipos, por ser este tipo el más extendido para dar soporte remoto con estos dispositivos, orientados sobre todo a alumnos de etapas educativas. También fue usada para valorar aplicaciones iOS y Android.

b. Líneas braille SuperVario: líneas de 40 caracteres con anotador. Usada especialmente por personas con sordoceguera.

c. Orbit 20: anotador con línea braille de 20 caracteres que puede utilizarse de manera autónoma como anotador o, conectado a dispositivos móviles u ordenadores, como línea braille.

2. Reproductores Daisy

a. Hardware: Victor Reader, Victor Stream, Milestone, PlexTalk. Todos ellos permiten la lectura de los libros en CD o descargados de la BDO.

b. Software: DaisyPlayer, Litersoft, AMIS, FS Reader. Instalados en un PC, reproducen los libros en formato Daisy de la BDO.

3. Lupas TV

a. Sobremesa: permiten visualizar en una pantalla, a través de una cámara (zoom), textos, objetos, imágenes que se depositan en la bandeja de lectura, pudiendo variarse la magnificación, los colores, los contrastes...

b. Manuales: de tamaño más reducido y fácil de transportar, facilitan la lectura o la visualización de objetos o imágenes por medio de la ampliación o el cambio de colores y contrastes. 


\section{Software}

1. Lectores de pantalla.

a. NVDA. Masivamente empleado en PC para formaciones, asistencia remota y apoyo de voz a la lupa de Windows o ZoomText.

b. JAWS. Instalado en PC, sobre todo en entornos educativos y laborales. También proporciona acceso remoto por medio de Tandem.

c. Narrador de Windows. Utilizado en formaciones remotas conectadas mediante otros lectores o para la instalación de ellos.

d. VoiceOver. Para Apple (iPhone, OsX y WatchOS). En las formaciones en la accesibilidad de esos dispositivos y también en la valoración de aplicaciones móviles. Consultas sobre Apple TV.

e. TalkBack. Para Android. Utilizado sobre todo en formaciones como complemento a la magnificación en Android o para valoraciones de aplicaciones móviles.

2. Magnificadores.

a. ZoomText. Instalado y configurado en varios ordenadores. Se pudo contar con licencias para acceso remoto, lo que facilitó el trabajo de los ITB y de los afiliados que teletrabajaban.

b. Lupa y características visuales de Windows. Ambas herramientas mejoran la visualización, configuradas sobre varios equipos.

3. Software para asistencias en remoto.

a. TeamViewer Corporativo. Software que permite realizar atenciones remotas en toda clase de dispositivos (iPhone, Android, PC y Mac). Se ampliaron las licencias para dar servicio a todos durante el confinamiento.

b. NVDA Remoto. Complemento que permite conexiones remotas sencillas a los usuarios de este lector de pantalla y el control de sus PC. 
c. JAWS Tandem. Dicha función, implementada en este lector de pantalla, permite atender directamente a usuarios de ese lector.

4. Software específico para educación y entretenimiento.

a. Edico. Software desarrollado por la ONCE para el aprendizaje de Matemáticas, Física y Química. Configurado para profesores y alumnos.

b. EBrai. Software desarrollado por la ONCE para la transcripción de textos a braille.

c. Mekanta y MIO: programas de mecanografía. Muchos afiliados adquirieron buenos hábitos de mecanografía durante el confinamiento.

d. Juegos educativos. Facilitado a maestros el acceso al variado contenido, junto a Tiflojuegos.

e. Videoteca Audesc y Biblioteca Digital ONCE.

f. Daisy Converter. Para aquellos usuarios que prefieren escuchar sus audiolibros en CD o MP3.

g. GOLD y Apolo. Las aplicaciones más demandas (en iOS y en Android) para el acceso a la BDO y a la Videoteca de la ONCE. Usado también el cliente de la BDO para enviar libros desde equipos Windows.

h. ClubONCE: acceso a la numerosa información específica creada para el momento de confinamiento, así como para la descarga de obras desde cualquier navegador, Windows o Mac.

5. Software OCR (siglas en inglés de reconocedor óptico de caracteres).

a. FineReader. Para convertir documentación poco accesible remitida a alumnos en etapas educativas.

b. OmniPage. Usado especialmente por afiliados en el entorno laboral para digitalizar documentación. 
C. OCR de JAWS. Usado cuando los requerimientos de la digitalización no son pesados.

d. Seeing AI, Voice Dream Scanner. Herramientas en dispositivos móviles que permiten el acceso a información escrita.

e. Lookout y Supersense: OCR portátil para teléfonos Android. 